\title{
Observation of complete inversion of the hysteresis loop in a bimodal magnetic thin film
}

\author{
Tuhin Maity, ${ }^{1, *}$ Demie Kepaptsoglou, ${ }^{2}$ Michael Schmidt, ${ }^{1}$ Quentin Ramasse, ${ }^{2}$ and Saibal Roy ${ }^{1, \dagger}$ \\ ${ }^{1}$ Tyndall National Institute, Lee Maltings, Dyke Parade, Cork, Ireland \\ ${ }^{2}$ SuperSTEM Laboratory, SciTech Daresbury Campus, Daresbury, United Kingdom \\ (Received 19 August 2016; revised manuscript received 1 February 2017; published 3 March 2017)
}

\begin{abstract}
The existence of inverted hysteresis loops (IHLs) in magnetic materials is still in debate due to the lack of direct evidence and convincing theoretical explanations. Here we report the direct observation and physical interpretation of complete IHL in $\mathrm{Ni}_{45} \mathrm{Fe}_{55}$ films with 1 to $2 \mathrm{~nm}$ thin $\mathrm{Ni}_{3} \mathrm{Fe}$ secondary phases at the grain boundaries. The origin of the inverted loop, however, is shown to be due to the exchange bias coupling between $\mathrm{Ni}_{45} \mathrm{Fe}_{55}$ and $\mathrm{Ni}_{3} \mathrm{Fe}$, which can be broken by the application of a high magnetic field. A large positive exchange bias $\left(H_{E B}=14 \times H_{C}\right)$ is observed in the $\mathrm{NiFe}$ composite material giving novel insight into the formation of a noninverted hysteresis loop (non-IHL) and IHL, which depend on the loop tracing field range $\left(H_{R}\right)$. The crossover from non-IHL to IHL is found to be at $688 \mathrm{Oe}$.
\end{abstract}

DOI: 10.1103/PhysRevB.95.100401

The hysteresis loop is a key characteristic of magnetic materials and depends on two main parameters, the remanence magnetization $\left(M_{R}\right)$ and the coercivity $\left(H_{C}\right)$ where $M_{R}$ and $H_{C}$ retain positive (negative) and negative (positive) values, respectively, for the descending (ascending) branch of the hysteresis loop and vice versa. This well-established characteristic was challenged by the observation of a so-called inverted hysteresis loop (IHL) where the loop is partially inverted in amorphous Gd-Co films [1]. This phenomenon was further observed in different magnetic systems, such as exchange-coupled multilayers [2-9], soft/hard magnetized materials [10], materials with two competing anisotropies, etc. [11-14]. Henceforth, the origin of the IHL has been investigated in different material systems, and several mechanisms/models based on different coupling effects, such as the magnetostatic interaction [3], exchange coupling [4], exchange spring, and competition of two anisotropies [12,13] have been proposed. Although a number of studies have been carried out, eventually it was realized that these hysteresis loops are "partially inverted," i.e., they exhibit noninverted behavior (anticlockwise) at higher fields and inverted (clockwise) behavior at lower fields or near the origin. The area within the hysteresis loop reflects the energy dissipated during the field cycle, and the negative area for the completely inverted loops would violate the first law of thermodynamics - a discrepancy that initially was explained through the so-called inhomogeneity effect $[2,15,16]$. Simultaneously theoretical models, such as wasp-waist hysteresis loops, the Preisach model of hysteresis, etc., were proposed [14,17-19]. Furthermore it was argued that inverted loops could arise from experimental artifacts rather than inhomogeneity [20]. Here we report the direct observation of a complete IHL in a NiFe thin film throughout the temperature range from room temperature $(300 \mathrm{~K})$ to low temperature $(5 \mathrm{~K})$ without any violation of thermodynamic principle. Most importantly this IHL is tunable to the noninverted hysteresis loop (non-IHL) and vice versa. Interestingly it has been observed that the formation

\footnotetext{
*Author to whom correspondence should be addressed: tuhin.maity@tyndall.ie

†saibal.roy@tyndall.ie
}

of these two opposite hysteresis loops depends upon the field range $\left(H_{R}\right)$ of hysteresis loop measurements. Furthermore we propose a model and interpretation for this observation.

In our Rapid Communication two different $\mathrm{Ni}_{45} \mathrm{Fe}_{55}$ thin films were prepared by electroplating [21]. The average grain size of $\mathrm{Ni}_{45} \mathrm{Fe}_{55}$ was found to be $\sim 15$ and $\sim 30 \mathrm{~nm}$ for two different samples prepared by modulated pulse reverse and constant direct current (dc) electroplating, respectively. Depending on the electrochemical bath composition, process parameters, and additives, dc electroplating produced thin films with an $\sim 30 \mathrm{~nm}$ grain size. Whereas, in a similar plating configuration by modulating the electrical wave form including repeated forward and reverse pulse cycles, a further reduction in grain sizes $(15 \mathrm{~nm})$ was achieved. We report here mainly the results obtained in samples with a $15 \mathrm{~nm}$ grain size. For a precise subnanoscale characterization, we carried out high-resolution transmission electron microscopy (HRTEM), atomic level scanning transmission electron microscopy (STEM) (see the Supplemental Material [21] for technical details), analysis, and electron energy loss spectroscopy (EELS) on an aberration-corrected Nion UltraSTEM. The results are summarized in Fig. 1 from which it is clear that the NiFe film consists of a polycrystalline granular $(10-30 \mathrm{~nm})$ ferromagnetic (FM) phase with an average film composition of $45 \% \mathrm{Ni}-55 \% \mathrm{Fe}$, along with a thin (1 to $2 \mathrm{~nm}$ ) secondary Fe deficient phase at the grain boundaries [blue boundary region in Fig. 1(d)] with the $\mathrm{Ni}$ and $\mathrm{Fe}$ ratio 3:1, likely to be $\mathrm{a} \mathrm{Ni}_{3} \mathrm{Fe}$ phase (for more details see the Structural characterization section in the Supplemental Material provided [21]). Both $\mathrm{Ni}_{45} \mathrm{Fe}_{55}$ and $\mathrm{Ni}_{3} \mathrm{Fe}$ are ferromagnetic, and the coercivity of the $\mathrm{Ni}_{3} \mathrm{Fe}$ nanograins is in the range of $\sim 20-80 \mathrm{Oe}$ (previously reported) higher than the coercivity (measured $H_{C}=0.5 \mathrm{Oe}$ ) of the bulk $\mathrm{Ni}_{45} \mathrm{Fe}_{55}$ alloy film [22-25]. Detailed magnetic measurements have been carried out in a superconducting quantum interference device (SQUID) magnetometer (MPMS XL5, Quantum Design) across a wide temperature range of 5-300 K under a maximum field $H_{\max }$ of $\pm 50 \mathrm{kOe}$. All precautions were taken to eliminate possible artifacts, if any [21].

Figure 2(a) shows a typical conventional exchange bias (CEB) measurement after cooling down from $350 \mathrm{~K}$ under a \pm 1000 Oe bias field. The hysteresis loops were measured with a loop tracing field range $\left(H_{R}\right)$ of \pm 200 Oe to ensure 


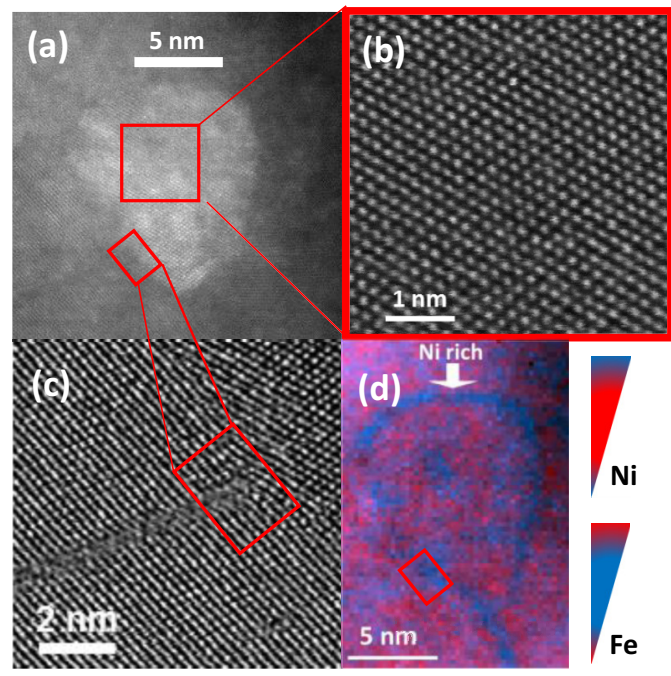

FIG. 1. Structural and elemental analyses of the NiFe film. (a) High angle annular dark-field (HAADF) STEM image of the NiFe film. The grain in the center of the image was aligned onto a [110] zone axis, and the brighter contrast observed, compared to the surrounding grain, is due to electron-beam channeling effects rather than a change in the chemistry of the grains. (b) Atomically resolved bright-field STEM image, showing details of the grain interior. The observed lattice fringes in the grain are consistent with FeNi (along a [110] crystal orientation) as also evidenced by spectroscopy measurements. (c) Lattice-resolved bright-field STEM image of the grain boundary, clearly showing the presence of an intergranular region distinct from the grains on either side. Here the lower left section of the outer grain is not aligned onto the same zone axis as the inner grain, and a dislocation appears to originate at the grain boundary (center of the red rectangle). (d) Composite chemical maps of this area are obtained by combining STEM-EELS maps for $\mathrm{Ni}$ and $\mathrm{Fe}$. The composite image was color coded for clarity, showing the presence of Ni-rich regions at the grain boundary. A more complete characterization is presented in the Supplemental Material section [21] provided.

magnetization saturation $\left(H_{S} \sim 50 \mathrm{Oe}\right)$ of the film. The region near the origin is magnified to show the extent of the exchange bias $\left(H_{E B}\right)$ clearly. The exchange bias observed here is quite large $H_{E B}= \pm 14$ Oe compared to its coercivity $\left(H_{C}\right)=1 \mathrm{Oe}$ measured at $5 \mathrm{~K}$. At $300 \mathrm{~K}$ the values are \pm 2.5 and 0.5 Oe, respectively (Fig. S6 in the Supplemental Material [21]). Depending on the sign of the polarity of the bias field $+1000 \mathrm{Oe} /-1000 \mathrm{Oe}$, the direction of the CEB is along a positive or negative field direction. Hence the exchange bias coupling at the interface is a positive exchange bias type (the loop shift is in the positive field direction when cooled down with the positive bias field and vice versa) in which the interfacial exchange interaction is believed to be antiferromagnetic (AFM). Furthermore we measured the hysteresis loop with a $\pm 50 \mathrm{kOe}$ field range $\left(H_{R}\right)$. The hysteresis loop appeared to be completely inverted with coercivity $\left|H_{C}\right| \sim 14 \mathrm{Oe}$, and no exchange bias was found where the loop saturates only at $\left|H_{S}\right| \sim 50$ Oe [Fig. 2(b)]. A surprising coincidence is that the coercivity $\left|H_{C}\right| \sim 14$ Oe for the IHL is almost equal to the exchange bias $\left|H_{E B}\right| \sim 14 \mathrm{Oe}$ of the non-IHL at $5 \mathrm{~K}$ where thermal perturbations are minimal and the exchange bias coupling is maximum. Neither a steplike distorted hysteresis (a)
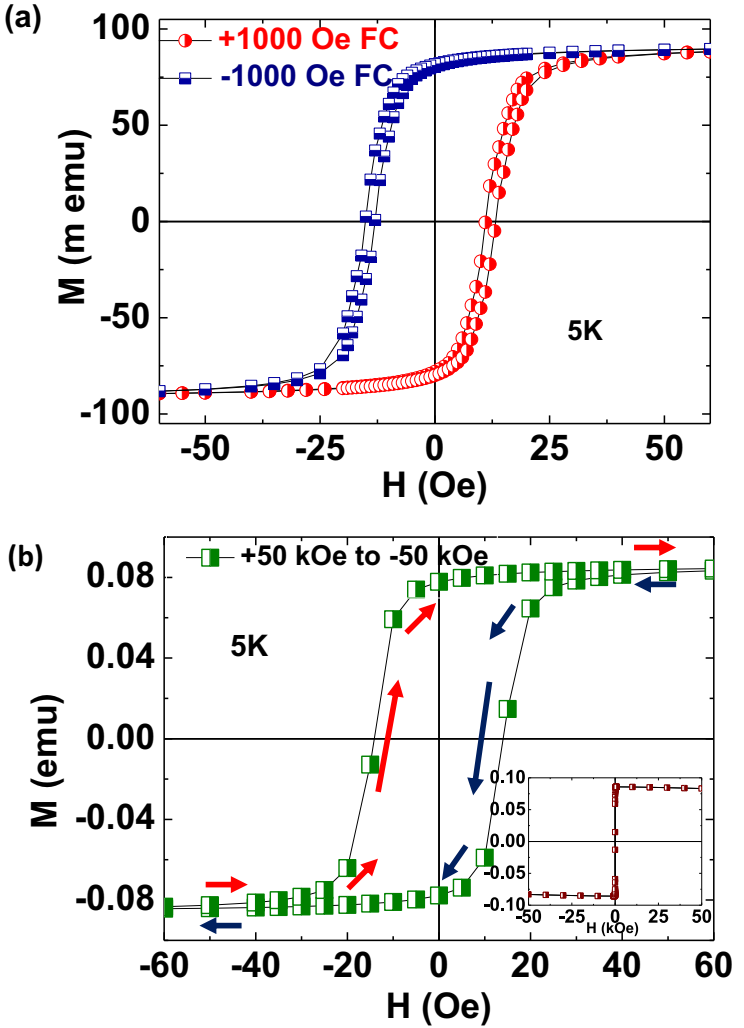

FIG. 2. Non-IHL and IHL formation (a) Conventional exchange bias measured at $5 \mathrm{~K}$ after cooling down from $350 \mathrm{~K}$ with a bias field of \pm 1000 Oe. Hysteresis loops were measured with a \pm 200 Oe field range $\left(H_{R}\right)$. The noninverted hysteresis loop was formed. (b) The hysteresis loop was measured with a \pm 50000 Oe field range $\left(H_{R}\right)$ at $5 \mathrm{~K}$. Complete IHL formation was observed. The inset figure shows that no steplike hysteresis loop was formed.

loop/helical loop [Fig. 2(b) inset] nor a crossover between ascending and descending loops was observed (see the Supplemental Material [21]). Both ascending and descending loops remain parallel and identical in magnetization values up to $50 \mathrm{kOe}$ when the loops saturate only at $\sim 50$ Oe [21].

For a clearer understanding, both the IHL (green $M H$ loop) and the non-IHL (blue and red loops) are plotted together in Fig. 3(a). It is observed that the descending and ascending branches of the IHL are parts of two different (positive and negative) exchange bias coupled non-IHLs with half $\delta M / \delta H$ strength and double in energy area compared to the non-IHL [the inset in Fig. 3(a)]. This indicates that the observed loop when measured with a $\pm 50 \mathrm{kOe}$ field is a pure inverted hysteresis loop where the "rate of approach to saturation" of magnetization is nearly half and it requires nearly double the amount of energy for the formation of the IHL compared to the non-IHL. Formation of the IHL is solely dependent on a bimodal exchange bias mechanism originating from $\mathrm{Ni}_{45} \mathrm{Fe}_{55}$ spins [the blue arrows in Fig. 3(b)] inside the grains and $\mathrm{Ni}_{3} \mathrm{Fe}$ spins [the red arrows in Fig. 3(b)] at the grain boundaries. The origin of the inverted hysteresis lies in the unique magnetic spin configuration at the grain boundary/interface which goes through complex but systematic changes depending on the 

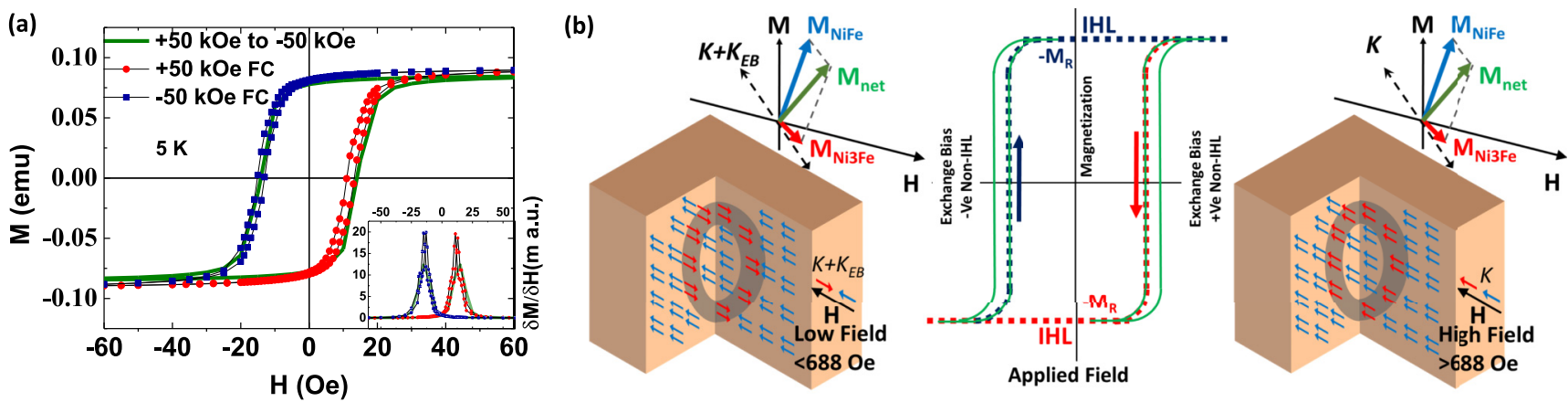

FIG. 3. Bimodal magnetic system for the IHL. (a) Ascending and descending branches of the IHL (the green lines) coincide with the negative (blue) and positive (red) non-IHL at $5 \mathrm{~K}$. The inset figure shows that variation of the magnetic moment $(\delta M / \delta H)$ as a function of the field which confirms that descending and ascending parts of the IHL originate from conventional positive and negative exchange bias loops, respectively. (b) The magnetic spin configurations at the low- and high-field regions are shown in the schematic due to the bimodal magnetic system for the IHL. The blue and red arrows denote magnetic spins of $\mathrm{Ni}_{45} \mathrm{Fe}_{55}$ and $\mathrm{Ni}_{3} \mathrm{Fe}$, respectively. $K$ is the uniaxial anisotropy of the thin film, and $K_{E B}$ is the exchange anisotropy which exists below the critical field $(<688 \mathrm{Oe}) . M_{\mathrm{NiFe}}, M_{\mathrm{Ni} 3 \mathrm{Fe}}$, and $M_{\mathrm{net}}$ are the magnetizations of $\mathrm{Ni}_{45} \mathrm{Fe}_{55}$, the $\mathrm{Ni}_{3} \mathrm{Fe}$ phases, and the net magnetization, respectively. During field reversal exchange bias coupling is generated at the grain interface due to coexistence of two different magnetic anisotropies (red and blue arrows) in opposite directions at the low field. The ascending (blue dashed line) and descending parts (red dashed line) of the IHL are originated from the ascending and descending branches of two conventional exchange bias loops (green continuous lines), respectively.

field applied and its history. This is summarized in the schematic in Fig. 3(b).

At high-field regimes both interfacial and core spins are aligned in the same direction. When the external magnetic field is reversed the exchange bias coupling at the interface is developed at low fields due to the antiparallel alignment of nonreversed $\mathrm{Ni}_{3} \mathrm{Fe}$ with the reversed $\mathrm{Ni}_{45} \mathrm{Fe}_{55}$ magnetization vector and spin pinning at the interface in the field reversal process. The volume fraction of $\mathrm{Ni}_{3} \mathrm{Fe}$ is extremely low $(\sim 1.5 \%)$ and thus is expected to have an insignificant contribution to the film's total magnetization [21,25]. Hence, the descending part of the IHL is generated at the positive field quadrant due to the positive exchange bias coupling at the interface of two different magnetic phases, and negative remanence magnetization $\left(-M_{R}\right)$ is obtained at a $H=0 \mathrm{Oe}$ field. The reciprocal mechanism is observed when the field is increased from a negative high field to a positive value and the ascending part of the IHL is generated in the negative field quadrant with positive remanence $\left(+M_{R}\right)$.

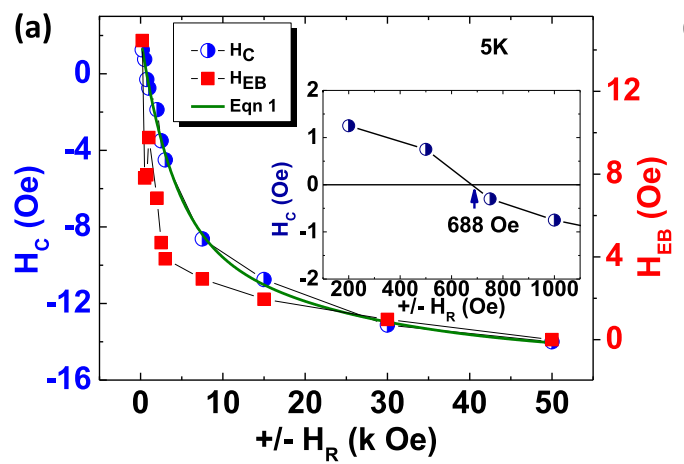

To find out whether the IHL depends on the applied field, the loop tracing range $\left(H_{R}\right)$ is gradually increased from \pm 200 to $\pm 50 \mathrm{kOe}$. The amount of exchange bias $\left(H_{E B}\right)$ and coercivity $\left(H_{C}\right)$ decreases up to a certain field [Fig. 4(a)]. Due to the increase in the loop tracing field, the exchange bias coupling generated at the interface during field reversal is broken gradually by the opposite high field and aligned in the field direction. Hence the amounts of exchange bias and coercivity are reduced. Due to the competition between the exchange-coupled energy at the interface and the anisotropy energy of the film after a certain field the loop switches to the other direction to minimize the system energy and the negative coercivity starts increasing in the negative direction where the exchange bias further decreases. The lowest exchange bias $\left(H_{\mathrm{EB}}=0 \mathrm{Oe}\right)$ and the highest negative coercivity $\left(H_{C}=\right.$ $14 \mathrm{Oe}$ ) are obtained with the loop tracing field range just below $\pm 50 \mathrm{kOe}$ at $5 \mathrm{~K}$ temperature. The inverted hysteresis loop or clockwise loop is observed only when the loop tracing field range is above \pm 688 Oe [Fig. 4(a)]. Below that value,

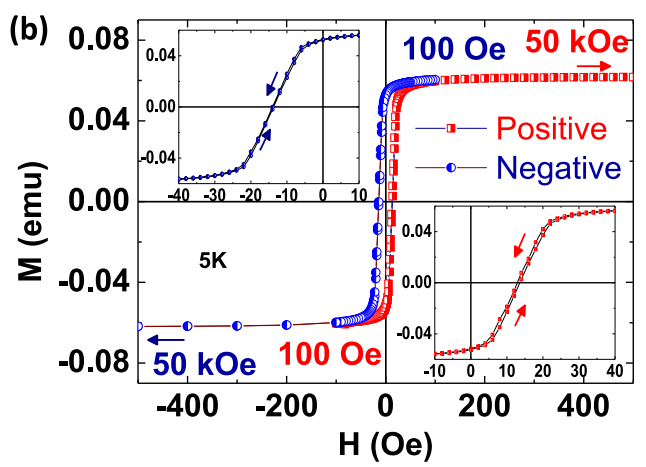

FIG. 4. Tunablity of the inverted hysteresis loop. (a) The hysteresis loop can be tuned from inverted to noninverted by changing the loop's tracing field range $\left(H_{R}\right)$. The demagnetization energy equation Eq. (1)] fits well with the variation of $H_{C}$ as a function of loops tracing the field range $\left(H_{R}\right)$. Above the 688 Oe field range the hysteresis loop is the IHL, and below that the non-IHL is observed. (b) The IHL does not form when measured with a high-to-low loop tracing field range $( \pm 50 \mathrm{kOe}>\mp 100 \mathrm{Oe}> \pm 50 \mathrm{kOe}$ or vise versa) at $5 \mathrm{~K}$. The inset figures show the paths followed by the non-IHL. 
the hysteresis loop is a usual anticlockwise or noninverted hysteresis loop. For the second sample of similar composition with a larger grain size of $30 \mathrm{~nm}$, the switching field was found to be 1750 Oe with similar IHL behavior. Therefore it seems that the switching field increases with the grain size. No IHL behavior was found in other $\mathrm{Ni}-\mathrm{Fe}$ alloys, such as $\mathrm{Ni}_{81} \mathrm{Fe}_{19}$.

Since the transition of the hysteresis loop from the IHL to the non-IHL is due to an exchange bias coupling, it is relevant to consider two different anisotropies $k_{\mathrm{FM}}$ and $k_{\mathrm{AFM}}$ for the interfacial spins. These two anisotropies behave differently at externally applied fields during the demagnetization process in the field reversal. A qualitative demagnetization energy equation,

$$
E=E_{0}+M_{\mathrm{FM}} e^{-\left(H / k_{\mathrm{FM}}\right)}+M_{\mathrm{AFM}} e^{-\left(H / k_{\mathrm{AFM}}\right)}
$$

fits the data very convincingly [the green line in Fig. 4(a)] (where $M_{\mathrm{FM}}, M_{\mathrm{AFM}}$ and $k_{\mathrm{FM}}, k_{\mathrm{AFM}}$ are the saturation magnetizations and relative anisotropies for ferromagnetic and antiferromagnetic phases, respectively) and yields the fitting parameters as $M_{\mathrm{FM}}=6.9, M_{\mathrm{AFM}}=9.7$ and $k_{\mathrm{FM}}=22646, k_{\mathrm{AFM}}=$ 3919. The ratio $k_{\mathrm{FM}} / k_{\mathrm{AFM}} \sim 5.8$ indicates that the field required to break the exchange bias coupling is $\sim 5.8$ times higher than the field required to rotate the uncoupled magnetic spins.

According to the Stoner-Wohlfarth model the energy per unit volume of such a magnetic system can be given by

$$
\begin{aligned}
E= & k_{\mathrm{AFM}} \sin ^{2} \theta_{\mathrm{AFM}}+k_{\mathrm{FM}} \sin ^{2} \theta_{\mathrm{FM}}-M_{\mathrm{AFM}} H \cos \left(\theta_{\mathrm{AFM}}-\phi\right) \\
& -M_{\mathrm{FM}} H \cos \left(\theta_{\mathrm{FM}}-\phi\right) \\
& -J_{E B} M_{\mathrm{AFM}} M_{\mathrm{FM}} \cos \left(\theta_{\mathrm{AFM}}-\theta_{\mathrm{FM}}\right) .
\end{aligned}
$$

The first two terms represent the uniaxial anisotropy energy, the second two terms represent the Zeeman energy of the AFM and FM phases, respectively, and the last term represents the exchange coupling between these two phases. $J$ is the antiferromagnetic exchange coupling constant. $\theta_{\mathrm{AFM}}$ and $\theta_{\mathrm{FM}}$ are the angles between $M_{\mathrm{AFM}}$ and $M_{\mathrm{FM}}$ with the easy axis of the $\mathrm{Ni}_{3} \mathrm{Fe}$ component, and $\phi$ is the angle between the applied field and the easy axis of the $\mathrm{Ni}_{3} \mathrm{Fe}$ component. At the saturation state, the total magnetization of $\mathrm{Ni}_{45} \mathrm{Fe}_{55}$ is much larger than that of $\mathrm{Ni}_{3} \mathrm{Fe}$ since the volume fraction of $\mathrm{Ni}_{3} \mathrm{Fe}$ is negligible $(\sim 1.5 \%)$.

Provided that the volume fraction of $\mathrm{Ni}_{3} \mathrm{Fe}$ is negligible and the applied field always is kept along the easy axis direction $(\phi=0)$ of the film, we can rewrite the equation,

$$
\begin{aligned}
E= & k_{\mathrm{AFM}} \sin ^{2} \theta_{\mathrm{AFM}}+k_{\mathrm{FM}} \sin ^{2} \theta_{\mathrm{FM}}-M_{\mathrm{FM}} H \cos \theta_{\mathrm{FM}} \\
& -J_{E B} M_{\mathrm{AFM}} M_{\mathrm{FM}} \cos \left(\theta_{\mathrm{AFM}}-\theta_{\mathrm{FM}}\right) .
\end{aligned}
$$

For the minimization of the system energy $\delta E / \delta \theta_{\mathrm{AFM}}$ and $\delta E / \delta \theta_{\mathrm{FM}}$ must be zero, and solutions for possible states (magnetic spin alignment) can be described by four approximate solutions: (i) $\theta_{\mathrm{AFM}}=\theta_{\mathrm{FM}}=0$, (ii) $\theta_{\mathrm{AFM}}=\pi$ and $\theta_{\mathrm{FM}}=0$, (iii) $\theta_{\mathrm{AFM}}=0$ and $\theta_{\mathrm{FM}}=\pi$, and (iv) $\theta_{\mathrm{AFM}}=\theta_{\mathrm{FM}}=\pi$. By examining the second derivatives of $E$, we find that the antiparallel spin alignment [states (ii) and (iii)] is the most stable state. However, for a sufficiently large applied field the identical states (i) and (iv) are not reversible due to the presence of exchange coupling at the interface. In other words, the state cannot be shifted from (i) to (iv) due to the existence of exchange bias coupling at the interface in the low-energy (/field) regime. Thus the reversible process is discontinued in a high-field relaxation process, a negative (positive) remanence is observed in the descending (ascending) loop, and an IHL is generated. Furthermore to confirm this exciting result and its origin, we measured the sample with a high starting field then reversed at the relatively lower (less than the critical field) field ( $\pm 50 \mathrm{kOe}>\mp 100 \mathrm{Oe}> \pm 50 \mathrm{kOe})$. A non-IHL with positive exchange bias was observed in this case since the exchange bias coupling was not broken by the lower reverse field [Fig. 4(b)].

In this particular NiFe system, two different ferromagnetic phases of $\mathrm{NiFe}$ with significantly different anisotropies, giving rise to antiferromagnetic (positive) exchange bias $\left(H_{E B}\right)$ greater than the coercivity $\left(H_{C}\right)$, are observed which provide suitable conditions for a complete IHL. It is thus apparent that for a pure IHL few criteria need to be fulfilled: (a) The exchange bias $\left(H_{E B}\right)$ should be positive in type, i.e., for a positive field, the loop shift should be in the positive direction; (b) the amount of exchange bias should be greater than the coercivity of the non-IHL $\left(H_{E B}>H_{C}\right)$; and (c) the secondary phase which contributes to the high exchange energy should have a negligible contribution in magnetization for the entire system. The first two criteria need to be fulfilled for the formation of an ascending (descending) loop in the positive (negative) field quadrant. The third criterion is essential for negative remanence and nonhelical/step hysteresis when the first two criteria already are fulfilled. If a system can fulfill these essential conditions, an IHL can be observed depending upon the magnetization process of both phases. Furthermore, the IHL can be tuned by balancing the energy and changing the relative contribution among the Zeeman energy, the anisotropy energy, and the exchange bias coupling energy under different fields in different energy regimes. Thus the observed inverted hysteresis loop with two different and distinct $H_{C}{ }^{\prime}$ s ( 1 and 14 Oe at $5 \mathrm{~K}$ ) in the same material and increased $B H_{\max }$ at above the critical field in this bimodal system can open the possibility for probing and manipulating the magnetic hysteresis loop, which could lead to paving new roads towards robust nanoscale micromagnetic device applications.

This Rapid Communication has been supported by the Science Foundation of Ireland (SFI) Principal Investigator (PI) Project No. 11/PI/1201 and the Irish Research Council Project No. GOIPD/2016/474. The SuperSTEM Laboratory is the United Kingdom's National Facility for Aberration-Corrected STEM, supported by the Engineering and Physical Sciences Research Council (EPSRC).
[1] S. Esho, Jpn. J. Appl. Phys. 15, 93 (1976).

[2] M. J. O'Shea and A. L. Al-Sharif, J. Appl. Phys. 75, 6673 (1994).
[3] A. Aharoni, J. Appl. Phys. 76, 6977 (1994).

[4] K. Takanashi, H. Kurokawa, and H. Fujimori, Appl. Phys. Lett. 63, 1585 (1993). 
[5] Y. Z. Wu, G. S. Dong, and X. F. Jin, Phys. Rev. B 64, 214406 (2001).

[6] S. M. Valvidares, L. M. Álvarez-Prado, J. I. Martín, and J. M. Alameda, Phys. Rev. B 64, 134423 (2001).

[7] S. Demirtas, M. R. Hossu, M. Arikan, A. R. Koymen, and M. B. Salamon, Phys. Rev. B 76, 214430 (2007).

[8] M. Ziese, I. Vrejoiu, and D. Hesse, Appl. Phys. Lett. 97, 052504 (2010).

[9] R. K. Zheng, H. Liu, Y. Wang, and X. X. Zhang, J. Appl. Phys. 96, 5370 (2004).

[10] D. Y. Kim, C. G. Kim, C.-O. Kim, S. S. Yoon, M. Naka, M. Tsunoda, and M. Takahashi, J. Magn. Magn. Mater. 304, e356 (2006).

[11] S. Ohkoshi, T. Hozumi, and K. Hashimoto, Phys. Rev. B 64, 132404 (2001).

[12] Y. J. Nam and S. H. Lim, Appl. Phys. Lett. 99, 092503 (2011).

[13] J. Geshev, A. D. C. Viegas, and J. E. Schmidt, J. Appl. Phys. 84, 1488 (1998).

[14] L. V. Tho, C. G. Kim, and C. O. Kim, J. Appl. Phys. 103, 07 B 906 (2008).
[15] Shuo Gu et al., Sci. Rep. 4, 6267 (2014)

[16] X. Yan and Y. Xu, J. Appl. Phys. 79, 6013 (1996)

[17] S. Bi, A. Sutor, R. Lerch, and Y. Xiao, IEEE Trans. Magn. 49 3175 (2013).

[18] L. H. Bennett and E. Della Torre, J. Appl. Phys. 97, 10E502 (2005).

[19] E. Della Torre, Magnetic Hysteresis (Wiley-IEEE, Piscataway, NJ,1998).

[20] C. Song, B. Cui, H. Y. Yu, and F. Pan, J. Appl. Phys. 114, 183906 (2013).

[21] See Supplemental Material at http://link.aps.org/supplemental/ 10.1103/PhysRevB.95.100401 for information on the methods.

[22] T. E. Cranshaw, J. Phys. F: Met. Phys. 17, 967 (1987).

[23] S. J. Yan, L. Zhen, C. Y. Xu, J. T. Jiang, and W. Z. Shao, J. Phys. D: Appl. Phys. 43, 245003 (2010).

[24] J. Y. Park and M. G. Allen, J. Micromech. Microeng. 8, 307 (1998).

[25] S. P. Li, S. Kulkarni, and S. Roy, J. Appl. Phys. 112, 103918 (2012). 